\title{
Differential benefit risk assessment of DOACs in the treatment of venous thromboembolism: focus on dabigatran
}

This article was published in the following Dove Press journal:

Drug Design, Development and Therapy

9 July 2015

Number of times this article has been viewed

\section{Timothy S Leung' \\ Ernest $\mathrm{H}$ Law $^{2}$}

'Faculty of Pharmaceutical Sciences, University of British Columbia, Vancouver, BC, Canada; ${ }^{2}$ Department of Pharmacy Systems, Outcomes and Policy, College of Pharmacy, University of Illinois at Chicago, Chicago, IL, USA

Correspondence: Timothy S Leung Faculty of Pharmaceutical Sciences, University of British Columbia, 2405 Wesbrook Mall, Vancouver, BC V6T IZ3, Canada

Email timleung@alumni.ubc.ca
Abstract: Venous thromboembolism includes deep vein thrombosis and pulmonary embolism and is a serious medical condition that requires anticoagulation as part of treatment. Currently, standard therapy consists of parenteral anticoagulation followed by a vitamin $\mathrm{K}$ antagonist (VKA). The pharmacokinetic and pharmacodynamic profiles of the direct oral anticoagulants (DOACs) differ from VKAs, which overcome some of the limitations of VKAs and have practical implications on their use in clinical situations. Dabigatran is a prodrug that undergoes primarily renal elimination and does not affect cytochrome P450 enzymes. Assays to quantify the degree of anticoagulation and the therapeutic level of DOAC are either unavailable for routine clinical use or require specific calibration. Routine monitoring of DOACs is not recommended at this time. Dabigatran, rivaroxaban, and apixaban are DOACs that have been studied for treatment of venous thromboembolism. Clinical trials comparing DOACs with standard therapy have shown them to be non-inferior for acute and extended therapy. Each DOAC has a unique benefit and harm profile that should be considered prior to use. The distinguishing characteristics of dabigatran include a requirement of parenteral anticoagulation prior to acute treatment, clinical trial results comparing it with a VKA for extended treatment, association with upper gastrointestinal adverse events, and increased risk of gastrointestinal bleed. Rivaroxaban is the only DOAC that has once-daily dosing while apixaban is the only DOAC that has lower risk of overall, major, and gastrointestinal bleeding compared with VKA. A common drawback of DOACs is the lack of an available reversal agent. Clinical trials of reversal agents are ongoing and one application for approval has been submitted to the US Food and Drug Administration. Selection of a DOAC for acute and extended therapy requires a shared decision-making approach that includes a comprehensive assessment of the benefits and harms of each individual DOAC.

Keywords: venous thromboembolism, dabigatran, anticoagulant, treatment

\section{Introduction}

Venous thromboembolism (VTE) is a condition that includes both deep vein thrombosis (DVT) and pulmonary embolism (PE). VTE may be provoked by identifiable clinical risk factors such as surgery, trauma, immobility, and cancer, or occur in the absence of these risk factors (unprovoked VTE). ${ }^{1}$ The rate of VTE is estimated to be up to 1.24 events per 1,000 person-years with a case-fatality rate of $14 \%$ at 30 days and $29 \%$ at 1 year. $^{2}$ Short-term complications of VTE may include pain and swelling of the affected leg in DVTs; and chest pain, dyspnea, and hypoxia in PEs. In the long term, complications include recurrent VTE, post-thrombotic syndrome, pulmonary hypertension, and death. ${ }^{1,3-5}$ To reduce recurrent VTE and mortality, current guidelines recommend acute and long-term anticoagulation with the duration of long-term therapy determined by whether the event was provoked or unprovoked and patient factors 
(eg, active cancer and history of VTE). ${ }^{6}$ In patients with acute $\mathrm{PE}$, current CHEST guidelines recommend acute treatment with low molecular weight heparin (LMWH) or intravenous unfractionated heparin and a vitamin $\mathrm{K}$ antagonist (VKA) for long-term anticoagulation. ${ }^{6}$ Prior to the development of direct oral anticoagulants (DOACs), frequent blood tests, drug-drug interactions, and drug-food interactions made patient adherence to VKA challenging. In contrast, DOACs such as dabigatran, rivaroxaban, and apixaban have several properties that may make these agents attractive alternatives in the treatment of VTE. In Canada and the USA, dabigatran is indicated for the treatment of VTE to reduce recurrent VTE. ${ }^{7,8}$ This review will discuss the pharmacology, clinical evidence, therapeutic issues, and the place in therapy of dabigatran in context of VTE treatment.

\section{Pharmacology, pharmacokinetics, and pharmacodynamics}

Dabigatran etexilate is a quickly absorbed prodrug with low bioavailability that is rapidly hydrolyzed into its active form (dabigatran) in the blood. ${ }^{9}$ Its anticoagulant effect is a result of direct inhibition of thrombin, which prevents formation of fibrin. ${ }^{7-9}$ A summary of the pharmacokinetic and pharmacodynamic parameters of dabigatran is presented in Table 1.

Relative to warfarin, there are fewer factors that affect the pharmacokinetic and pharmacodynamic profile of dabigatran.
Food has no effect on the bioavailability of dabigatran; however, coadministration with food increases the time to maximum plasma concentration $\left(\mathrm{T}_{\max }\right)$ from 2 to 4 hours but does not affect maximum plasma concentration $\left(\mathrm{C}_{\max }\right)$ or area under the curve (AUC). ${ }^{6}$ Administration of dabigatran to patients who underwent total hip replacement increased the $\mathrm{T}_{\max }$ of dabigatran to 6 hours in 1 day following surgery. ${ }^{10}$ Bioavailability may be increased 1.8-fold if contents of the capsule alone are taken. ${ }^{7}$

AUC may be decreased by $20 \%$ in patients weighing greater than $120 \mathrm{~kg}$ and increased by $25 \%$ in patients weighing less than $48 \mathrm{~kg}$; however, no recommendation for dosage adjustment is provided by the product monograph or CHEST guidelines. ${ }^{7}$ Concomitant use of a proton-pump inhibitor can also reduce $\mathrm{C}_{\max }$ and decrease AUC by $20 \%-25 \% .^{9-11}$ The clinical relevance of this interaction is uncertain and although the Health Canada (HC) monograph recommends caution, it does not provide a recommendation for dosage adjustment. The US Food and Drug Administration (FDA) monograph makes no comment on this issue. ${ }^{8}$

In addition to its predominantly renal elimination, dabigatran has properties that suggest amenability to removal by extracorporeal methods. Dabigatran is a low molecular weight anticoagulant with a volume of distribution mostly limited to the blood compartment, and has low protein binding.

Table I Differential pharmacology of DOACs

\begin{tabular}{|c|c|c|c|}
\hline & Dabigatran & Rivaroxaban & Apixaban \\
\hline Bioavailability & $6.5 \%$ & $100 \%$ b & $50 \%$ \\
\hline $\mathrm{T}_{\max }$ & 2 hours $^{\mathrm{a}}$ & $2-4$ hours & $3-4$ hours \\
\hline Half-life $\left(t_{1 / 2}\right)$ & $12-14$ hours & 7-II hours & 8 hours \\
\hline Volume of distribution & $60-70 \mathrm{~L}$ & $50 \mathrm{~L}$ & $21 \mathrm{~L}$ \\
\hline Protein binding & $35 \%$ & $92 \%-95 \%$ & $87 \%-93 \%$ \\
\hline \multirow[t]{2}{*}{ Metabolism } & Hydrolysis to active form & CYP3A4, 2J2 & CYP3A4/5 (major) \\
\hline & & CYP-independent mechanisms & CYPIA2, 2C8, 2C9, 2CI9, 2J2 (minor) \\
\hline \multirow[t]{3}{*}{ Elimination } & $80 \%-85 \%$ renal as unchanged drug & $33 \%$ renally as unchanged drug & $25 \%$ renally as unchanged drug \\
\hline & $20 \%$ biliary & $33 \%$ renally as inactive metabolite & $55 \%$ in feces as inactive metabolite \\
\hline & & $33 \%$ in feces as inactive metabolite & $20 \%$ via other mechanisms \\
\hline \multirow[t]{10}{*}{ Selected drug interactions } & P-gp inhibitors & Ketoconazole & Ketoconazole \\
\hline & P-gp inducers & Ritonavir & Rifampin \\
\hline & Antiplatelets & Rifampicin & Antiplatelets \\
\hline & Anticoagulants & Clarithromycin & Anticoagulants \\
\hline & NSAIDs & Antiplatelets & NSAIDs \\
\hline & Amiodarone & Anticoagulants & \\
\hline & Verapamil & NSAIDs & \\
\hline & & Phenytoin & \\
\hline & & Carbamazepine & \\
\hline & & Phenobarbital & \\
\hline Selected food interactions & None & None & None \\
\hline
\end{tabular}

Notes: aProlonged if administered on the day of surgery. ${ }^{b}$ When taken with food. Data from studies. ${ }^{8-12}$

Abbreviations: CYP, cytochrome P450; DOACs, direct oral anticoagulants; NSAID, nonsteroidal anti-inflammatory drug; P-gP, P-glycoprotein. 
Both forms of dabigatran (prodrug and active form) lack cytochrome P450 interactions. However, the prodrug dabigatran etexilate is a P-glycoprotein (P-gp) substrate. ${ }^{7,8}$

The anticoagulant effects of dabigatran mirror plasma concentrations; therefore, maximum effect $\left(\mathrm{E}_{\max }\right)$ coincides with $\mathrm{C}_{\max }$ and $\mathrm{T}_{\max }$ reflects time to $\mathrm{E}_{\max } \cdot{ }^{9}$ The onset of action of dabigatran is fast and can be expected to be approximately 2 hours after administration. ${ }^{7}$ After one half-life $\left(t_{1 / 2}\right)$, the maximum effect of dabigatran is reduced by $30 \% .{ }^{9}$ Antiplatelet agents (eg, aspirin and clopidogrel) have no direct effects on the anticoagulant activity of dabigatran, though concurrent use of these agents increases bleeding risk.

Dabigatran can affect multiple coagulation assays; however, the clinical significance of these methods have yet to be clarified, making the routine use of these tests inadvisable until further evidence is available. ${ }^{12}$ Furthermore, not all tests may be readily available for routine clinical use. Even when available, the timing of the blood sample, patient factors (eg, coadministration with food, drug interactions, and renal function), and equipment/reagent used further affect the results and interpretation. ${ }^{13,14}$ Dabigatran has a curvilinear relationship with activated partial thromboplastin time (aPTT), which is not sensitive enough to quantify the degree of anticoagulation. ${ }^{13}$ As such, aPTT may be useful as a qualitative indicator of dabigatran effect and a trough level taken 12-24 hours after a dose may suggest an excess bleeding risk if it is greater than two times the upper limit of normal. ${ }^{15}$ Ecarin clotting time (ECT) and diluted thrombin time $\left(\mathrm{dTT}, \mathrm{Hemoclot}^{\mathbb{}}\right)$ provide a more direct measure of anticoagulation but ECT is currently restricted only to research settings and dTT may not be readily available in all clinical settings. ${ }^{15,16}$ Dabigatran can also falsely elevate prothrombin time (PT) and international normalized ratio (INR), which reduces the clinical utility of these results. ${ }^{7,8,15,16}$

In comparison, factor Xa inhibitors (ie, rivaroxaban and apixaban) affect PT differently and both have variable effects on INR, aPTT, dTT, and ECT. A prolonged/elevated PT while on rivaroxaban may indicate an excess bleeding risk but calibration of the equipment and reagent must be performed before interpretation. ${ }^{15}$ In contrast, PT does not provide clinically relevant information about apixaban. ${ }^{15}$ There is evidence to suggest that anti-Xa activity may have a linear relationship with both rivaroxaban and apixaban if appropriate calibration and reagents are used. ${ }^{12,17,18}$

Except in select clinical scenarios (eg, suspicion of DOAC use in life-threatening bleed), routine use of coagulation assays for managing anticoagulation with a DOAC is not recommended due to a lack of evidence linking assay results with clinical outcomes. ${ }^{12,15}$

\section{Clinical trials}

The use of DOACs in management of VTE has been evaluated in several settings. DOACs have been compared with standard therapy (parenteral anticoagulants and a VKA) for acute therapy and compared with warfarin and placebo for long-term treatment. Summaries of major clinical trials investigating the use of DOACs for acute and extended therapy in VTE are provided in Tables 2 and 3, respectively. ${ }^{19-25}$

\section{RE-COVER and RE-COVER II}

The first clinical trial comparing a DOAC with standard therapy for treatment of acute VTE was the RE-COVER. ${ }^{19}$ RECOVER was a randomized, double-blind, double-dummy, non-inferiority trial that compared dabigatran $150 \mathrm{mg}$ twice daily with warfarin with a target INR of 2.0-3.0 in the setting of acute VTE. All patients received a course of parenteral anticoagulation prior to receiving the study drug. Patients were similar at baseline and followed for 13 months. Long-term antiplatelet therapy was not permitted except for concomitant aspirin, provided that the total daily dose was $\leq 100 \mathrm{mg}$. The number of patients who were on concomitant aspirin was not reported. The primary outcome was a composite of symptomatic VTE and death due to VTE. Major bleeding was classified according to the International Society of Thrombosis and Haemostasis (ISTH) guidelines and defined as fatal bleeding and/or symptomatic bleeding in a critical area or organ and/or a fall in hemoglobin level of $20 \mathrm{~g} / \mathrm{L}$ or more or leading to transfusion of two or more units of whole blood or red cells. ${ }^{26}$ Minor bleeding was defined as less severe bleeds or nuisance bleeding. To achieve noninferiority, the upper bound of the hazard ratio (HR) for the primary outcome had to be less than 2.75 and achieve an absolute risk increase (ARI) of less than $3.6 \%$, which corresponds to $57 \%$ and $75 \%$ of the lower bound of the confidence interval (CI) in previous warfarin trials, respectively. At the end of the study, the margin for non-inferiority was reached (HR 1.10, 95\% CI 0.65-1.84 and ARI 0.3\%, 95\% CI $0.8-1.5)$. Major bleeding was similar between both groups but when pooled with clinically relevant nonmajor bleeds, the number of bleeds in the dabigatran arm was significantly lower (HR 0.63, 95\% CI 0.47-0.84). There was no difference in the number of discontinuations between the two arms. The time in therapeutic range (TTR) or proportion of INR values maintained between 2.0 and 3.0 was $60 \%$ and adherence was $98 \%$ in the study.

The RE-COVER II trial utilized the same design as RECOVER and was performed to extend the findings. ${ }^{20}$ Patients in both studies were similar except for a greater proportion of 
Table 2 Summary of clinical trials of DOACs for acute treatment of VTE

\begin{tabular}{|c|c|c|c|c|c|}
\hline & RE-COVER & RE-COVER II & EINSTEIN-PE & EINSTEIN-DVT & AMPLIFY \\
\hline Intervention ${ }^{\mathrm{a}}$ & $\begin{array}{l}\text { Parenteral } \\
\text { anticoagulation } \\
\text { (median } 9 \text { days), then } \\
\text { dabigatran } 150 \mathrm{mg} \text { bid }\end{array}$ & $\begin{array}{l}\text { Parenteral } \\
\text { anticoagulation } \\
\text { (median } 9 \text { days), then } \\
\text { dabigatran } 150 \mathrm{mg} \text { bid }\end{array}$ & $\begin{array}{l}\text { Rivaroxaban } 15 \mathrm{mg} \\
\text { bid for } 21 \text { days, } \\
\text { then } 20 \mathrm{mg} \text { daily }\end{array}$ & $\begin{array}{l}\text { Rivaroxaban } 15 \mathrm{mg} \\
\text { bid for } 21 \text { days, then } \\
20 \mathrm{mg} \text { daily }\end{array}$ & $\begin{array}{l}\text { Apixaban } 10 \mathrm{mg} \\
\text { bid for } 7 \text { days, } \\
\text { then } 5 \mathrm{mg} \text { bid }\end{array}$ \\
\hline Comparator & \multicolumn{2}{|c|}{$\begin{array}{c}\text { LMWH, then warfarin dosed to target INR } \\
\text { within } 2.0-3.0\end{array}$} & \multicolumn{3}{|c|}{ Warfarin dosed to target INR within 2.0-3.0 } \\
\hline Duration of $T x$ & 6 months & 6 months & 3,6 , or 12 months & 3,6 , or 12 months & 6 months \\
\hline No of patients & 2,539 & 2,568 & 4,832 & 3,449 & 5,395 \\
\hline Mean age & 55 years & 56 years & 57 years & 56 years & 57 years \\
\hline Renal function & $\begin{array}{l}\text { Mean } \mathrm{CrCl} \mathrm{Fl} \\
\sim 105 \mathrm{~mL} / \mathrm{min}\end{array}$ & $\begin{array}{l}\text { Mean } \mathrm{CrCl} \\
\sim 108 \mathrm{~mL} / \mathrm{min}\end{array}$ & \multicolumn{2}{|c|}{ Majority were $>50 \mathrm{~mL} / \mathrm{min}$} & $\begin{array}{l}\text { Majority were } \\
>50 \mathrm{~mL} / \mathrm{min}\end{array}$ \\
\hline DVT only & $69 \%$ & $68 \%$ & N/A & $99 \%$ & $65 \%$ \\
\hline DVT and PE & $10 \%$ & $9 \%$ & $25 \%$ & $\mathrm{~N} / \mathrm{A}$ & $9 \%$ \\
\hline Unprovoked VTE & NR & NR & $64 \%$ & $62 \%$ & $90 \%$ \\
\hline Prior VTE & $25 \%$ & $18 \%$ & $20 \%$ & $19 \%$ & $16 \%$ \\
\hline Malignancy & $5 \%$ & $4 \%$ & $5 \%$ & $6 \%$ & $2 \%$ \\
\hline Thrombophilia & $N R$ & $N R$ & $5 \%$ & $6 \%$ & $2 \%$ \\
\hline \multirow[t]{2}{*}{ Primary outcome ${ }^{a}$} & Dabigatran: $2.4 \%$ & Dabigatran: $2.3 \%$ & Rivaroxaban: $2.1 \%$ & Rivaroxaban: $2.1 \%$ & Apixaban: $2.3 \%$ \\
\hline & Standard Tx: $2.1 \%$ & Standard Tx: $2.2 \%$ & Standard Tx: $1.8 \%$ & Standard Tx: $3.0 \%$ & Standard Tx: $2.7 \%$ \\
\hline \multirow[t]{2}{*}{ Major bleeding ${ }^{\mathrm{b}}$} & Dabigatran: I.6\% & Dabigatran: I.2\% & Rivaroxaban: I.I\% & Rivaroxaban: $0.8 \%$ & Apixaban: $0.6 \%$ \\
\hline & Standard Tx: $1.9 \%$ & Standard Tx: $1.7 \%$ & Standard Tx: $2.2 \%$ & Standard Tx: $1.2 \%$ & Standard Tx: $1.8 \%$ \\
\hline Clinically relevant & Dabigatran: $4.0 \%$ & Dabigatran: $3.8 \%$ & Rivaroxaban: $9.2 \%$ & Rivaroxaban: $7.3 \%$ & Apixaban: $3.8 \%$ \\
\hline bleeding & Standard Tx: $6.9 \%$ & Standard Tx: $6.2 \%$ & Standard Tx: $9.2 \%$ & Standard Tx: $7.0 \%$ & Standard Tx: $8 \%$ \\
\hline $\begin{array}{l}\text { Time in therapeutic } \\
\text { range for INR }\end{array}$ & $60 \%$ & $57 \%$ & $63 \%$ & $58 \%$ & $61 \%$ \\
\hline
\end{tabular}

Notes: aRE-COVER, RE-COVER II: Symptomatic VTE and death; EINSTEIN-PE, EINSTEIN-DVT: recurrent, symptomatic VTE; AMPLIFY: recurrent symptomatic VTE or associated with VTE. ${ }^{b}$ As per the International Society of Thrombosis and Haemostasis guidelines. Data from studies. ${ }^{20,21,23-25}$

Abbreviations: DOACs, direct oral anticoagulants; DVT, deep vein thrombosis; INR, international normalized ratio; LMWH, low molecular weight heparin; N/A, not applicable; NR, not reported; PE, pulmonary embolism; VTE, venous thromboembolism; Tx, therapy.

Table 3 Summary of clinical trials of DOACs for extended treatment of VTE

\begin{tabular}{|c|c|c|c|c|}
\hline & RE-MEDY & RE-SONATE & EINSTEIN-EXT & AMPLIFY-EXT \\
\hline Intervention & Dabigatran $150 \mathrm{mg}$ bid & Dabigatran $150 \mathrm{mg}$ bid & Rivaroxaban 20 mg daily & Apixaban $2.5 \mathrm{mg}$ or $5 \mathrm{mg}$ daily \\
\hline Comparator & $\begin{array}{l}\text { Warfarin (INR } \\
2.0-3.0)\end{array}$ & Placebo & Placebo & Placebo \\
\hline Duration of Tx & 36 months & 30 months & 265 days & 12 months \\
\hline No of patients & 2,866 & $\mathrm{I}, 353$ & 1,196 & 2,482 \\
\hline Mean age & 54 years & 55 years & 58 years & 56 years \\
\hline Renal function & NR & $N R$ & Majority were $>50 \mathrm{~mL} / \mathrm{min}$ & Majority were $>50 \mathrm{~mL} / \mathrm{min}$ \\
\hline DVT only & $65 \%$ & $65 \%$ & NR & NR \\
\hline DVT and PE & $12 \%$ & $6 \%$ & NR & $N R$ \\
\hline Malignancy & $4 \%$ & - & $5 \%$ & $1.7 \%$ \\
\hline Thrombophilia & $18 \%$ & $12 \%$ & $8 \%$ & NR \\
\hline \multirow[t]{2}{*}{ Primary outcome ${ }^{\mathrm{a}}$} & Dabigatran: $1.8 \%$ & Dabigatran: $0.4 \%$ & Rivaroxaban: I.3\% & Apixaban 2.5 mg daily: $3.8 \%^{\mathrm{d}}$ \\
\hline & Warfarin: $1.3 \%$ & Placebo: $5.6 \%$ & Placebo: $7.1 \% c$ & $\begin{array}{l}\text { Apixaban } 5 \mathrm{mg} \text { daily: } 4.2 \%^{\mathrm{d}} \\
\text { Placebo: } 1 \mathrm{I} .6 \%\end{array}$ \\
\hline \multirow[t]{2}{*}{ Major bleeding ${ }^{\mathrm{b}}$} & Dabigatran: $0.9 \%$ & Dabigatran: $0.3 \%$ & Rivaroxaban: $0.7 \%$ & Apixaban 2.5 mg daily: $0.2 \%$ \\
\hline & Warfarin: $1.8 \%$ & Placebo: $0 \%$ & Placebo: $0 \%$ & $\begin{array}{l}\text { Apixaban } 5 \mathrm{mg} \text { daily: } 0.1 \% \\
\text { Placebo: } 0.5 \%\end{array}$ \\
\hline Clinically relevant & Dabigatran: $4.7 \%$ & Dabigatran: $5 \%$ & Rivaroxaban: $5.4 \%$ & Apixaban $2.5 \mathrm{mg}$ daily: $3.0 \%$ \\
\hline \multirow[t]{2}{*}{ nonmajor bleeding } & Warfarin: $8.4 \%$ & Placebo: $1.8 \%$ & Placebo: $1.2 \%$ & Apixaban $5 \mathrm{mg}$ daily: $4.2 \%$ \\
\hline & & & & Placebo: $2.3 \%$ \\
\hline $\begin{array}{l}\text { Time in therapeutic } \\
\text { range for INR }\end{array}$ & $65 \%$ & $\mathrm{~N} / \mathrm{A}$ & $N / A$ & $\mathrm{~N} / \mathrm{A}$ \\
\hline
\end{tabular}

Notes: aRE-MEDY, RE-SONATE: recurrent symptomatic VTE or death associated with VTE; EINSTEIN-EXT: recurrent, symptomatic VTE; AMPLIFY-EXT: recurrent, symptomatic VTE or death from any cause. ${ }^{b}$ As per the International Society of Thrombosis and Haemostasis guidelines. ${ }^{27}$ 'Some patients had more than one event. ¿Statistically significant difference compared with placebo. Data from studies. ${ }^{22-26}$

Abbreviations: DOACs, direct oral anticoagulants; DVT, deep vein thrombosis; INR, international normalized ratio; LMWH, low molecular weight heparin; N/A, not applicable; NR, not reported; PE, pulmonary embolism; VTE, venous thromboembolism; Tx, therapy. 
Asian patients in RE-COVER II (21\% vs 2\%). The reduction in recurrent VTE and death associated with VTE in RECOVER II was almost identical to RE-COVER (HR 1.08, 95\% CI $0.64-1.80$ and ARI $0.2 \%, 95 \%$ CI 1.0-1.3). As with RE-COVER, there was no difference in major bleeding; however, the composite of major and clinically relevant nonmajor bleeding was statistically significantly higher in the warfarin arm. A pooled analysis of the results from 5,107 patients in both RE-COVER and RE-COVER II showed that dabigatran maintained non-inferiority with respect to the primary outcome (HR 1.09, 95\% CI 0.76-1.57) and no increase in major bleeding. Major bleeding and clinically relevant nonmajor bleeding was lower in the dabigatran arm (HR 0.62, 95\% CI 0.50-0.76). A post hoc analysis of the results according to age showed no significant difference in efficacy but an increase in bleed risk with dabigatran in patients $>85$ years. Adherence to study medication was high (98\%) and TTR was 57\%. Both RE-COVER and RE-COVER II were designed, conducted, and funded by the manufacturer of dabigatran, who also analyzed the data for both trials.

\section{RE-MEDY and RE-SONATE}

The use of dabigatran for prevention of recurrent symptomatic VTE was studied in the RE-MEDY and RE-SONATE, both of which were randomized, double-blind trials. RE-MEDY was a non-inferiority trail comparing dabigatran $150 \mathrm{mg}$ twice daily with warfarin targeting an INR of between 2.0 and 3.0. Patients were similar at baseline except for a greater number of patients with diabetes mellitus and hypertension in the dabigatran arm. All patients were to have completed at least 3 months of VTE treatment and were followed for 36 months. The primary outcome was recurrent VTE or death associated with VTE. The definitions of major and clinically relevant nonmajor bleeding were the same as that in RE-COVER and RE-COVER II. To achieve non-inferiority to warfarin, the $\mathrm{HR}$ and the ARI for the primary outcome had to be less than $2.75 \%$ and $2.8 \%$, respectively, at 18 months. Both margins were determined based on rates of VTE in previous trials. With respect to the primary outcome, dabigatran was noninferior to warfarin (HR 1.44, 95\% CI 0.78-2.64). Major bleeding occurred at similar rates between both arms but major or clinically relevant bleeding occurred more often in the warfarin arm (HR 0.52, 95\% CI 0.27-1.02). TTR was $65 \%$ of the time and adherence was $98 \%$ in this trial.

RE-SONATE was a superiority trial comparing dabigatran with placebo. ${ }^{21}$ At baseline, patients were similar except for more hypertension in the dabigatran arm. After completing a minimum of 3 months of VTE treatment, patients were randomized to dabigatran or placebo and followed for
30 months. As with RE-MEDY, the primary outcome was recurrent VTE or death associated with VTE and definitions of major and clinically relevant nonmajor bleeding were the same as that in RE-COVER and RE-COVER II. Dabigatran was found to be superior to placebo (HR 0.08, 95\% CI $0.02-0.25)$ for the primary outcome. No major bleeding events occurred in the placebo arm while two occurred in the dabigatran arm. A statistically significant increase in major or clinically relevant bleeding was seen in the dabigatran arm (HR 2.92, 95\% CI 1.52-5.60). As with RE-COVER and RE-COVER II, both RE-MEDY and RE-SONATE were designed, conducted, and funded by the manufacturer of dabigatran, who also analyzed the data for both trials.

Clinical trials for acute VTE treatment with dabigatran show non-inferiority to warfarin with a similar rate of major bleeding but lower risk of clinically relevant nonmajor bleeding. Extended treatment of VTE with dabigatran demonstrated non-inferiority to warfarin and superiority compared with placebo. With extended treatment of VTE with dabigatran, clinically relevant nonmajor bleeding was lower when compared with warfarin but higher when compared with placebo. Major bleeding was similar between dabigatran and warfarin Patients with $\mathrm{CrCl}<30 \mathrm{~mL} / \mathrm{min}$ were excluded from clinical trials, while patients with active cancer were underrepresented. As such, CHEST guidelines do not recommend acute treatment with dabigatran for more than 6 months and advise against use in patients with active cancer. However, since publication of the current CHEST guidelines, RE-MEDY and RE-SONATE have been completed and results are available. They suggest that extended therapy in patients who would otherwise be on warfarin may be candidates for dabigatran. A summary of clinical trials of DOACs for extended treatment of VTE can be found in Table 3.

\section{Safety and tolerability}

An important safety concern with dabigatran is the risk of bleeding. Since dabigatran has anticoagulant effects that correlate directly with plasma concentrations, reduced renal function and certain drug interactions can increase the risk of bleeding by increasing exposure to dabigatran. Dosage adjustments can be considered with reduced renal function and the elderly who have reduced renal function and an increased risk for bleeding (see "Dosing and administration"). While patients up to 97 years old were included in all dabigatran trials, the average age of patients was approximately 55 years old. Therefore, bleeding rates in the trials may not sufficiently represent rates expected in elderly patients, given the decline in renal function with age. Another potential risk factor identified by a systematic review is female sex. ${ }^{27}$ The results 
of this systematic review are limited by the availability of sex-stratified data from DOAC trials (dabigatran VTE trials did not include such data) and the unclear clinical impact of this risk factor.

\section{Bleeding definition and overall bleed risk}

All clinical trials evaluating DOACs defined major bleeding according to ISTH critera. ${ }^{26}$ However, the definition of clinically relevant nonmajor bleeding differed between trials of dabigatran and trials of rivaroxaban or apixaban. In general, major bleeding was defined as overt bleeding not classified as major or requiring medical intervention or unscheduled meeting with a physician. In the rivaroxaban and apixaban trials, if a patient interrupted or stopped the study drug or it caused discomfort or impaired activities of daily life, then the event was classified as clinically relevant nonmajor bleeding. In the dabigatran trials, any bleeding event not classified as major could be considered a minor bleed if the investigator felt it was relevant. The risk of any bleeds or combined major and clinically relevant nonmajor bleeding with dabigatran was statistically significantly lower when compared with standard therapy in acute and extended VTE treatment. ${ }^{20,21}$ A similar result was seen with apixaban for acute VTE treatment; data for this outcome were not provided in the EINSTEIN and EINSTEIN-PE trials. ${ }^{22-24}$

\section{Major bleeding}

A recent systematic review and meta-analysis investigated the risk of major bleeding with DOACs compared with VKAs for VTE treatment or stroke and systemic embolism prophylaxis in atrial fibrillation (AF). ${ }^{28}$ The primary outcome was major bleeding as defined by the ISTH guidelines. ${ }^{26}$ Compared with VKA, there was a lower risk of major bleeding with the DOACs (relative risk [RR] 0.72, 95\% CI 0.62-0.85). Analysis of the subgroup of patients on DOACs for VTE treatment also resulted in a lower risk of major bleeding compared with LMWH followed by VKA. Sensitivity analysis that excluded unblinded studies did not change the results. In clinical trials, there was no statistically significant difference in major bleeding episodes between dabigatran and standard therapy nor was there a difference between rivaroxaban and standard therapy when treating DVT. Both rivaroxaban, when used in the treatment of acute PE, and apixaban, for acute VTE treatment, had fewer major bleeding episodes than warfarin.

\section{Intracranial hemorrhage}

Intracranial hemorrhage (ICH) is a life-threatening complication of anticoagulant therapy. Warfarin therapy is associated with an increased risk of death and disability due to $\mathrm{ICH}{ }^{29,30}$ In both RE-COVER and RE-COVER II, the absolute number of ICH episodes was lower in the dabigatran group compared with standard therapy. In EINSTEIN, the number of ICH was not presented but the rivaroxaban arm and standard therapy arm had the same number of critical site bleeds. EINSTEIN-PE had fewer episodes of ICH than the standard therapy group. Similarly, in AMPLIFY, fewer patients in the apixaban arm experienced ICH compared with patients on standard therapy. Patients on DOACs for non-valvular AF also had a lower incidence of $\mathrm{ICH} .{ }^{31-33}$ A recent systematic review and meta-analysis found that compared to standard therapy with a LMWH and VKA, DOACs had a significantly lower risk of ICH in patients treated for VTE; however, this was conducted as a subgroup analysis. ${ }^{28}$

\section{Gastrointestinal bleeding}

Another complication of anticoagulation is gastrointestinal bleeding (GIB). The absolute number of episodes of GIB in RE-COVER and RE-COVER II was higher in patients taking dabigatran than those taking warfarin. Neither EINSTEIN nor EINSTEIN-PE reported episodes of GIB. Patients on apixaban in the AMPLIFY trial had fewer GIB than those on warfarin. GIB was more common in patients on dabigatran and those on rivaroxaban in their respective AF trials; apixaban was not found to have significantly more GIB compared with warfarin in $\mathrm{AF}^{31-33}$ Holster et al evaluated the risk of GIB and clinically relevant bleeding in patients taking DOACs for various indications. ${ }^{34}$ The risk of a GIB was higher with DOACs compared with standard care (OR 1.45, 95\% CI 1.07-1.97) but there was significant heterogeneity among the studies included. When results of studies on DOACs for VTE treatment were meta-analyzed, there was a statistically significant increase in GIB risk in patients on DOACs (OR 1.59, 95\% CI 1.03-2.44) with low statistical heterogeneity. The results of AMPLIFY were not included as the trial was not published when the review was performed. However, a more recent systematic review and meta-analysis found in a subgroup analysis that patients on DOACs for VTE treatment had a lower risk for GIB (RR 0.64, 95\% CI 0.41-0.99). ${ }^{28}$ Taken together, these results suggest that each DOAC has a different effect on risk of GIB, with dabigatran increasing risk of GIB.

\section{Reversal agent}

At this time, a reversal agent for dabigatran is not available for clinical use. Idarucizumab, a monoclonal antibody (aDabi-Fab), is structurally similar to thrombin but without 
any coagulant activity. It has an affinity for dabigatran that is 350 times stronger than dabigatran's affinity for thrombin, allowing idarucizumab to neutralize the activity of dabigatran at a steady-state concentration of $200 \mathrm{ng} / \mathrm{mL}$ within 1 minute. ${ }^{35}$ Effects of aDabi-Fab were maintained for 25 minutes despite continuous addition of dabigatran. Conference abstracts of small clinical trials of idarucizumab in healthy, elderly, and renally impaired adults suggest that the antidote is effective and safe. ${ }^{36,37}$ Patients undergoing emergency procedures requiring reversal of dabigatran are being enrolled for a phase III, open-label, case series clinical trial with idarucizumab (Clinicaltrials.gov identifier: NCT02104947). The manufacturer of idarucizumab has applied for accelerated approval procedure with HC, FDA, and the European Medicines Agency. ${ }^{38}$

\section{Non-bleeding upper gastrointestinal adverse events}

Dyspepsia was more common in patients taking dabigatran in both RE-COVER $(3.1 \%$ vs $0.7 \%, P<0.001)$ and RE-COVER II $(1.0 \%$ vs $0.2 \%) .{ }^{19,20}$ While the incidence of dyspepsia was not reported in either RE-SONATE or RE-MEDY, one would expect higher rates compared with warfarin or placebo given the results of other trials with dabigatran which have consistently reported higher rates of dyspepsia compared with warfarin. ${ }^{31}$ Details regarding the types of non-bleeding upper gastrointestinal (NB-UGI) adverse events (AE) are provided by an analysis of data from the 18,113 patients in the RE-LY trial. ${ }^{39}$ The majority of patients with NB-UGI AE rated their symptom severity as mild to moderate (with a similar distribution of severity in patients on warfarin). Patients who were taking dabigatran were more likely to discontinue the drug than those on warfarin (4.0\% vs $1.7 \%$, RR 2.34, 95\% CI 1.90-2.88), regardless of dose and most often within the first 3 months of therapy. Female sex, age of at least 75 years old, Caucasian race, use of proton-pump inhibitor, use of histamine-2 receptor antagonist, use of nonsteroidal anti-inflammatory drug (NSAID), and use of a cyclooxygenase II inhibitor were independently associated with NB-UGI AE. The differing rates of dyspepsia between patients in RE-LY compared with RE-COVER and RE-COVER II, despite similar doses of dabigatran used, suggest underlying clinical differences between patients being treated for VTE and AF.

\section{Acute coronary syndromes}

There has been a concern with the risk of acute coronary syndrome (ACS) in patients receiving dabigatran since results of the RE-LY trial revealed a higher rate of ACS in patients on dabigatran compared with warfarin. ${ }^{31}$ Additional metaanalyses have supported the association of dabigatran with increased rates of ACS; however, these analyses have typically included clinically heterogeneous populations, making it difficult to determine which patients may be at the higher risk for ACS events. ${ }^{40,41}$ While there is no pharmacological or physiological mechanism to completely explain this risk associated with dabigatran, there is evidence that suggests that warfarin may be protective against ACS. ${ }^{42}$ This supports the hypothesis that the increase in ACS risk reflects a loss of this protective effect with warfarin rather than a direct effect of dabigatran. Therefore, in patients who have recently experienced an ACS event and develop VTE, the use of warfarin may be prudent.

Patients who require treatment for VTE may have comorbid conditions that require dual antiplatelet therapy (DAPT), such as ACS. However, there is little evidence to guide management of anticoagulation with DOACs for VTE in these patients. Three trials compared DOACs with placebo in the context of recent ACS and DAPT and found an increased risk of bleeding with the combination of DOAC and DAPT. ${ }^{43-45}$ Therefore, it is reasonable to expect an increase in bleeding in patients receiving DAPT. Clinicians should view this as an opportunity to reevaluate the patient's bleeding risk and indication for DAPT. In patients with high risk of bleeding who still require anticoagulation and DAPT, warfarin may be the preferred anticoagulant given the availability of a reliable reversal agent should a bleed occur.

\section{Therapeutic issues and controversy}

In patients who present with a bleed and suspected dabigatran use, a prolonged aPTT and TT can confirm the presence of dabigatran. Anti-Xa levels may be useful in determining whether the patient is on rivaroxaban or apixaban but the results depend on appropriate calibration of reagents and assays. Without a reliable and available antidote for DOACs, management of bleeding in these patients centers around discontinuation of the DOAC and other offending agents and providing supportive care. ${ }^{46}$

Since dabigatran undergoes primarily renal excretion, maintaining adequate global and regional perfusion, and ensuring adequate urine output may help to avoid prolonged anticoagulation in life-threatening bleeds. Based on a $t_{1 / 2}$ of 12-14 hours for dabigatran in a patient with normal renal function, approximately $75 \%$ of the previous dose of dabigatran will have been eliminated from the body after 24 hours. Near-complete elimination of the dose will take 
five $t_{1 / 2}$, or approximately 72 hours. The physicochemical properties of dabigatran suggest that it may be eliminated by dialysis. A review of evidence for removal of dabigatran by extracorporeal methods by Awad et al concluded that while certain clinical scenarios (such as emergent surgery and life-threatening bleeding) may mandate emergent access for dialysis, discordant evidence on whether dabigatran redistributes after dialysis and bleeding risks in establishing central access in an anticoagulated patient limit dialysis as a routine strategy. ${ }^{47}$ Whether redistribution occurs is important as the movement of dabigatran from tissue back into blood may necessitate further dialysis. The relatively low renal elimination of rivaroxaban and apixaban suggests that they will not be adequately removed by dialysis. Removal by this method may have a place in emergencies but has not been evaluated prospectively in clinical trials. The use of hemostatic agents such as prothombin complex concentrates, factor VIII inhibitor bypassing activity, and recombinant factor VIIa have not been studied in clinical trials in patients on DOACs requiring emergent reversal and should be considered only in select patients. ${ }^{48}$

DOACs may affect several coagulation assays but unlike warfarin, a single, clinically available assay that accurately reflects the degree of anticoagulation is unavailable. Dabigatran can be directly measured and an analysis of data collected from over 12,000 patients who were enrolled in the RE-LY trial evaluated the relationship between steady-state trough concentration (drawn 10-16 hours after a dose) and risk of bleed and ischemic event. ${ }^{49}$ The variation in plasma concentration was approximately fivefold among patients regardless of whether they were on dabigatran $110 \mathrm{mg}$ twice daily or $150 \mathrm{mg}$ twice daily. Trough concentrations correlated proportionally with dose. Most (80\%) patients achieved a trough concentration between $28-155$ and $40-215 \mathrm{ng} / \mathrm{mL}$ for the $110 \mathrm{mg}$ twice daily and $150 \mathrm{mg}$ twice-daily dosing, respectively. Higher trough concentrations were associated with higher bleed risk and lower concentrations associated with higher ischemic event risk. Both age and renal function were predictors of higher trough concentrations, which is not surprising since dabigatran is primarily renally eliminated and renal function declines with age. Given that higher trough concentrations are associated with an increased bleed risk, older patients and patients with reduced renal function may benefit from measurement of dabigatran trough concentrations to determine whether they are at higher risk of bleeding.

\section{Dosing and administration}

Dosing of dabigatran for the treatment of VTE in patients with $\mathrm{CrCl}>30 \mathrm{~mL} / \mathrm{min}$ is the same in both Canada and the USA. Dosing recommendations are summarized in Table 4. In patients with a $\mathrm{CrCl}>30 \mathrm{~mL} / \mathrm{min}, \mathrm{HC}$ and the $\mathrm{FDA}$ recommend 5-10 days of parenteral anticoagulation prior to starting dabigatran for the treatment and prevention of secondary VTE. ${ }^{7,8}$ This mirrors the protocol used in the RE-COVER and RE-COVER II trials. ${ }^{19,20}$ While neither recommendation specifies a particular number of days, the mean duration of parenteral anticoagulation in both trials was approximately 9 days. This should be taken into consideration along with patient factors when determining total duration of parenteral anticoagulation.

$\mathrm{HC}$ and the FDA monographs have different recommendations for dose adjustment in patients with reduced renal function. The HC monograph does not make a strong recommendation on dosage adjustment when $\mathrm{CrCl}$ is $30-50 \mathrm{~mL} / \mathrm{min}$ but suggests a dose of $110 \mathrm{mg}$ twice daily as a consideration. ${ }^{7}$ In contrast, the monograph from the FDA only makes a distinction if $\mathrm{CrCl}<30 \mathrm{~mL} / \mathrm{min}^{8}{ }^{8}$ These are a reflection of the clinical trials with dabigatran, which excluded patients with $\mathrm{CrCl}$ of $<30 \mathrm{~mL} / \mathrm{min}$. The recommendation to consider dabigatran $110 \mathrm{mg}$ twice-daily dosing in VTE treatment in patients with a $\mathrm{CrCl}$ of $30-50 \mathrm{~mL} / \mathrm{min}$ is based on pharmacokinetic data as this regimen has not been studied in clinical trials. ${ }^{7}$

Table 4 Dosing recommendations for dabigatran

\begin{tabular}{lll}
\hline Clinical scenario & Health Canada & FDA \\
\hline $\mathrm{CrCl} \geq 30 \mathrm{~mL} / \mathrm{min}$ & $150 \mathrm{mg}$ twice daily & $150 \mathrm{mg}$ twice daily \\
$\mathrm{CrCl}<30 \mathrm{~mL} / \mathrm{min}$ & $110 \mathrm{mg}$ twice daily & - \\
$\mathrm{CrCl} \mathrm{I5-30} \mathrm{mL/min}$ & Contraindicated & $75 \mathrm{mg}$ twice daily \\
$\mathrm{Age} \geq 80$ years old & $110 \mathrm{mg}$ twice daily & Not available \\
Age $<80$ years old & $150 \mathrm{mg}$ twice daily & Not available \\
Age $\geq 75$ years old and I risk factor for bleed* & $110 \mathrm{mg}$ twice daily & Not available \\
\hline
\end{tabular}

Notes: *Risk factors: $\mathrm{CrCl}$ 30-50 mL/min, concomitant P-gP inhibitor, NSAID use, antiplatelet use, coagulation disorders, thrombocytopenia, active gastrointestinal ulcer, recent gastrointestinal bleed, recent biopsy or major trauma, recent intracranial hemorrhage, brain/spinal/ophthalmic surgery, and bacterial endocarditis. Data from studies. ${ }^{8,9}$ Abbreviations: FDA, Food and Drug Administration; NSAID, non-steroidal anti-inflammatory drug; P-gp, P-glycoprotein. 
Increased exposure to dabigatran and increased risk of bleeding should be expected with P-gp inhibitors coadministration while a reduction in anticoagulant effect with concomitant use of P-gp inducers should be anticipated. Dabigatran therapy is contraindicated in patients receiving a strong P-gp inhibitor; dose adjustment is recommended for other P-gp inhibitors and inducers. ${ }^{7,8}$

Conversion between a VKA and dabigatran requires consideration of the anticipated duration of anticoagulation effect of each agent to minimize the risk of over or under anticoagulation. When transitioning a patient from a VKA to dabigatran, avoiding excessive anticoagulation by waiting until the INR is less than 2.0 prior to starting dabigatran is recommended. ${ }^{78}$ For patients converting from dabigatran to warfarin, a balance of time to elimination of dabigatran and the slow onset of effect of VKAs should be considered. For patients with $\mathrm{CrCl}>50 \mathrm{~mL} / \mathrm{min}$, it is recommended to start the VKA $\geq 3$ days prior to stopping dabigatran, while starting 2 days prior to stopping dabigatran in patients with $\mathrm{CrCl} 30-50 \mathrm{~mL} / \mathrm{min}$ should be sufficient in most situations. ${ }^{7}$ Clinicians should be aware that dabigatran can falsely elevate the INR; thus the effect of the VKA on the INR will become apparent 2 days after discontinuing dabigatran. ${ }^{7,8}$

Similar considerations are required when starting or stopping a parenteral anticoagulant. However, the shorter onset and offset of these agents allow dabigatran to be started 0-2 hours prior to discontinuing the parenteral anticoagulant and starting a parenteral anticoagulant 12 hours after stopping dabigatran. ${ }^{7,8}$

\section{Place in therapy}

The decision to start anticoagulation and the subsequent choice of anticoagulant should involve a comprehensive assessment of the individual clinical parameters, potential benefits and harms of therapy, and incorporation of patient values. Shared decision-making with the patient is important to guide therapy, as there is a wide variation in patient values and preferences in choosing anticoagulation for VTE management. ${ }^{50}$ The lack of head-to-head trials between DOACs in the current literature makes direct comparisons difficult, stressing the importance of shared and informed decision making when choosing a specific DOAC for anticoagulation. A proposed algorithm for choosing an agent for acute VTE treatment is shown in Figure 1.

An important issue when interpreting the results of clinical trials comparing DOACs with standard therapy (VKA with and without parenteral anticoagulation) for acute treatment of VTE is that these studies were typically non-inferiority. A more thorough discussion of this trial design is beyond the scope of this review; however, put simply, demonstrating non-inferiority can be interpreted as concluding that an intervention is "no worse than" standard therapy. ${ }^{51,52}$ The clinician and the patient should be aware that these clinical trials are not designed to support DOACs as being equivalent or superior to standard therapy. Non-inferiority trials set an arbitrary limit, or non-inferiority margin, where the observed effect of the comparator may be no less than the observed effect of standard therapy to be deemed non-inferior. Clinicians and patients must decide whether this potential decrease in efficacy outweighs the practical benefit(s) of the drug (eg, convenience and adverse effects). While all DOACs met their respective non-inferiority criteria, the loss in efficacy may be 1.18-1.57 times less than the comparator used, depending on the DOAC.

Extended treatment of VTE with DOACs has been studied for treatment durations between 12 and 36 months..$^{21,22,25}$ While all of the DOACs demonstrated a statistically significant reduction in recurrent VTE after patients had been on acute treatment for at least 6 months, only dabigatran has been studied against an active comparator (warfarin) and not placebo. In addition, data are available for up to 36 months of extended treatment with dabigatran. Dabigatran, rivaroxaban, and $2.5 \mathrm{mg}$ daily apixaban had lower rates of bleeding relative to their comparator; bleeding rates were higher with apixaban $5 \mathrm{mg}$ daily.

Compared with standard therapy, DOACs have the advantage of convenience in that blood tests are not required for routine management of therapy and there are fewer interactions with food and other drugs. Patients who value a lower risk of ICH with the DOACs and a lower risk of clinically relevant nonmajor bleeding may prefer dabigatran or apixaban.

Dabigatran has several unique disadvantages compared with rivaroxaban and apixaban. Choosing dabigatran as an anticoagulant for acute VTE treatment necessitates a treatment course with a parenteral anticoagulant, reflecting the protocol of RE-COVER and RE-COVER II. Patients received a median of 9 days (minimum 5 days) of parenteral anticoagulation prior to starting dabigatran. Based on the rapid onset of dabigatran, one could consider starting dabigatran without parenteral anticoagulation. However, uncertainty remains about the efficacy and safety of this strategy since it has not been studied in clinical trials. Clinical practice may preclude the use of dabigatran for acute treatment, since a course of parenteral anticoagulation prior to starting dabigatran might be unacceptable or impractical. In contrast, rivaroxaban and 


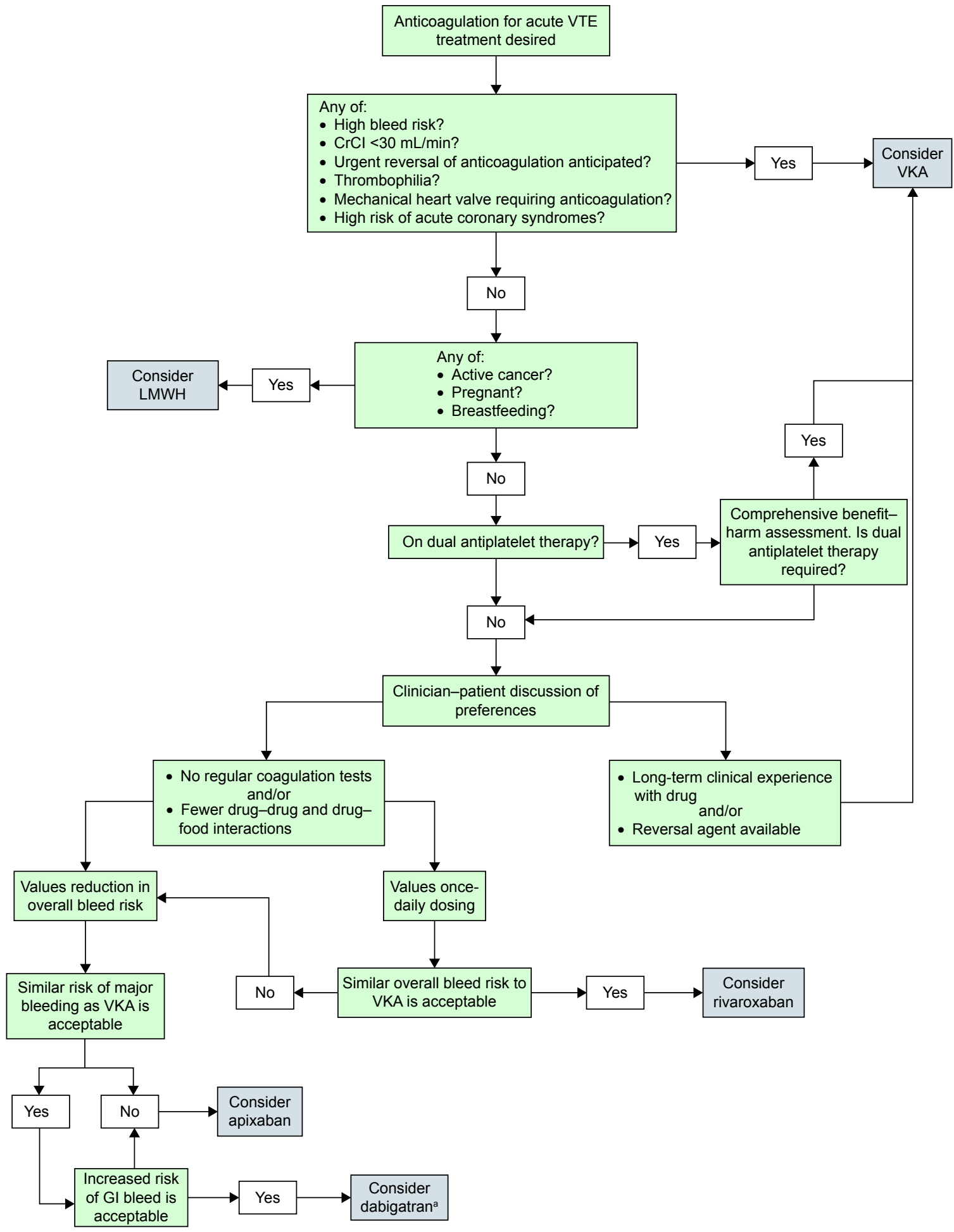

Figure I Proposed algorithm for DOAC selection in acute treatment for VTE.

Notes: ${ }^{a}$ Do not consider if minimum 5 days parenteral anticoagulation prior to dabigatran is unacceptable/impractical.

Abbreviations: DOACs, direct oral anticoagulants; GI, gastrointestinal; LMWH, low molecular weight heparin; VKA, vitamin K antagonist; VTE, venous thromboembolism.

apixaban were studied without a previous parenteral anticoagulation regimen and may be started when the choice of anticoagulant is made. Careful attention should be paid to the regimens studied, since rivaroxaban and apixaban used higher initial doses.
A drawback to use dabigatran is the higher incidence of NB-UGI AE. While not typically considered life-threatening, it may be burdensome enough that patients discontinue therapy. Extrapolation of data in patients on dabigatran for AF suggests that most patients who discontinue therapy 
due to NB-UGI AE while on dabigatran for VTE treatment will do so within 3 months of starting therapy. The same data suggest that female patients over the age of 75 , on an NSAID or cyclooxygenase II inhibitor, or on acid suppression therapy are more likely to experience NB-UGI AE. Exact management of NB-UGI AE is unclear but suggested strategies include taking dabigatran with food or two glasses of water and avoiding a supine position for at least 30 minutes after administration..$^{39}$ Ongoing symptoms despite management attempts may prompt consideration of other etiologies. ${ }^{39}$

While available evidence suggests that DOACs have a lower incidence of bleeding compared with standard therapy, patients and clinicians should be aware that the risk for GIB differs among individual DOACs. Specifically, dabigatran appears to carry an increased risk of GIB relative to warfarin. In contrast, apixaban had a lower incidence of GIB compared with warfarin. Data on GIB for rivaroxaban are not available. Based on this, dabigatran may not be the preferred choice in patients in who are at increased risk of GIB or place higher value on avoiding GIB.

A commonly raised concern surrounding DOACs is the current lack of an available reversal agent, which makes management of bleeding and preparation for emergency procedures potentially more difficult. Coagulation factors have been effective in reversing coagulation markers for rivaroxaban in healthy subjects but not for dabigatran. ${ }^{53}$ The use of coagulation factors has not been studied in clinical trials; therefore, mainstays in management are discontinuation of anticoagulation and supportive care while waiting for the patient to clear the drug. ${ }^{48}$ Phase III trials for idarucizumab for dabigatran reversal and andexanet alfa for rivaroxaban and apixaban are underway (Clinicaltrials.gov identifier NCT02104947, NCT02329327, NCT02220725, and NCT02207725). Compounding this issue is the lack of a reliable and readily available assay to measure the degree of anticoagulation.

In patients who require anticoagulation for acute VTE treatment, similar efficacy between the DOACs and VKA place much of the decision on other factors. Patients who prefer treatments that have "stood the test of time" that also have a readily available reversal agent may decide that treatment with a VKA is most suitable. However, these patients should also accept regular blood tests and be aware of multiple drug and food interactions. Those who place value on convenience in the form fewer blood tests and interactions may opt for a DOAC. The specific DOAC depends on many factors such as bleed risk, tolerability, and dosing frequency, which have been discussed in this review. The overall bleed risk of each DOAC differs relative to VKA. Both dabigatran and apixaban have lower risk while rivaroxaban is similar to VKA. Major bleeding is lower with apixaban but similar to VKA with dabigatran and rivaroxaban. No difference was seen with respect to GIB or NB-UGI AE with apixaban or rivaroxaban; dabigatran had higher rates of both. Rivaroxaban has the advantage of once-daily dosing. Dabigatran may be preferred in patients having an indication for long-term anticoagulation treatment for greater than 12 months and would otherwise require VKA therapy.

DOACs may be an attractive option to some patients requiring treatment for acute VTE but certain patients may not be candidates for DOACs. The proportion of patients with active cancer in acute VTE treatment trials ranged from $2 \%$ to $6 \%$. Results from a recent systematic review and metaanalysis evaluating the efficacy and safety of DOACs in patients with VTE and cancer suggest that DOACs appear to be similar to conventional therapy. ${ }^{54}$ However, conventional therapy with LMWH should be considered before DOACs in these patients until clinical trials with patients appropriately randomized to treatment arms are performed. Standard therapy should be considered in patients with thrombophilia, since the proportion in clinical trials was either not reported (RE-COVER, RE-COVER II, and AMPLIFY-EXT) or made up a minority of total patients $(2 \%-5 \%$ in EINSTEIN, EINSTEIN-PE, and AMPLIFY; $12 \%-18 \%$ in RE-MEDY and RE-SONATE). Based on exclusion criteria in clinical trials, patients who should not be treated with DOACs include those who are pregnant or breastfeeding and those with a $\mathrm{CrCl}<30 \mathrm{~mL} / \mathrm{min}$. The teratogenicity in humans with DOACs is unknown and patients with renal failure have an excessively high risk of bleeding because of an inability to effectively eliminate DOACs.

\section{Summary}

Standard anticoagulation therapy for treatment of acute VTE includes a parenteral anticoagulant followed by a VKA. DOACs are an attractive alternative due to their less frequent monitoring, relatively predictable pharmacokinetics, and fewer drug and food interactions. Evidence supports DOACs as an alternative in certain patients based on similar efficacy to VKAs and less bleeding overall.

The requirement of prior course of parenteral anticoagulation with dabigatran provides a significant challenge to its place as a viable option for acute VTE treatment. Its place in extended therapy may be clearer since only dabigatran was studied against an active comparator. In addition, trial data for dabigatran extend to 36 months, compared with 12 months for rivaroxaban and apixaban. Rivaroxaban has the advantage 
of once-daily dosing after an initial twice-daily dosing regimen, while apixaban carries a lower risk of major and clinically relevant nonmajor bleeding and is not associated with an increased risk of GIB relative to warfarin.

The efficacy and the safety of DOACs for VTE treatment in specific populations remain in question, due to either underrepresentation or exclusion from clinical trials. Specifically, there is a lack of evidence for the use of DOACs in patients who have active cancer, moderate renal function, thrombophilia, are on DAPT, or are pregnant or breastfeeding.

Based on differences in risk of AEs, pharmacokinetics, and clinical trial design among the DOACs, shared decisionmaking between the patient and the clinician can aid in selection of the most appropriate anticoagulant. As clinical experience is acquired and evidence becomes available, the place in therapy of DOACs for treatment of VTE should become clearer.

\section{Acknowledgment}

Ernest H Law was supported by 2014-2016 UIC/Takeda Fellowship in Health Economics and Outcomes Research.

\section{Disclosure}

The authors report no conflicts of interest in this work.

\section{References}

1. Wells PS, Forgie MA, Rodger MA. Treatment of venous thromboembolism. JAMA. 2014;311(7):717-728.

2. Tagalakis V, Patenaude V, Kahn SR, Suissa S. Incidence of and mortality from venous thromboembolism in a real-world population: the Q-VTE Study Cohort. Am J Med. 2013;126(9):832.e13-e21.

3. Middeldorp S, Prins MH, Hutten BA. Duration of treatment with vitamin K antagonists in symptomatic venous thromboembolism. Cochrane Database Syst Rev. 2014;8:CD001367.

4. Kahn SR, Ginsberg JS. Relationship between deep venous thrombosis and the postthrombotic syndrome. Arch Intern Med. 2004;164(1): 17-26.

5. Pengo V, Lensing AWA, Prins MH, et al. Incidence of chronic thromboembolic pulmonary hypertension after pulmonary embolism. $N \mathrm{Engl}$ J Med. 2004;350(22):2257-2264.

6. Kearon C. Antithrombotic therapy for VTE disease. Chest. 2012;141 (2 Suppl):e419S.

7. Boehringer Ingelheim Ltd. PRADAXA Monograph (Canada). 2014: $1-64$.

8. Boehringer Ingelheim Ltd. PRADAXA Monograph (US). 2014: $1-24$.

9. Eriksson BI, Quinlan DJ, Weitz JI. Comparative pharmacodynamics and pharmacokinetics of oral direct thrombin and factor xa inhibitors in development. Clin Pharmacokinet. 2009;48(1):1-22.

10. Stangier J, Eriksson BI, Dahl OE, et al. Pharmacokinetic profile of the oral direct thrombin inhibitor dabigatran etexilate in healthy volunteers and patients undergoing total hip replacement. J Clin Pharmacol. 2005; 45(5):555-563.

11. Stangier J, Stähle H, Rathgen K, Fuhr R. Pharmacokinetics and pharmacodynamics of the direct oral thrombin inhibitor dabigatran in healthy elderly subjects. Clin Pharmacokinet. 2008;47(1):47-59.
12. Cuker A, Siegal DM, Crowther MA, Garcia DA. Laboratory measurement of the anticoagulant activity of the non-vitamin K oral anticoagulants. J Am Coll Cardiol. 2014;64(11):1128-1139.

13. van Ryn J, Stangier J, Haertter S, et al. Dabigatran etexilate - a novel, reversible, oral direct thrombin inhibitor: interpretation of coagulation assays and reversal of anticoagulant activity. Thromb Haemost. 2010; 103(6):1116-1127.

14. Stangier J. Clinical pharmacokinetics and pharmacodynamics of the oral direct thrombin inhibitor dabigatran etexilate. Clin Pharmacokinet. 2008;47(5):285-295.

15. Heidbuchel H, Verhamme P, Alings M, et al. European Heart Rhythm Association Practical Guide on the use of new oral anticoagulants in patients with non-valvular atrial fibrillation. Europace. 2013;15(5): 625-651.

16. Liew A, Eikelboom JW, O'Donnell M, Hart RG. Assessment of anticoagulation intensity and management of bleeding with old and new oral anticoagulants. Can J Cardiol. 2013;29(7):S34-S44.

17. Bayer Inc. Xarelto Product Monograph (Canada). 2014:1-74.

18. Pfizer Inc. Eliquis Product Monograph (Canada). 2014:1-66.

19. Schulman S, Kearon C, Kakkar AK, et al. Dabigatran versus warfarin in the treatment of acute venous thromboembolism. N Engl J Med. 2009; 361(24):2342-2352.

20. Schulman S, Kakkar AK, Goldhaber SZ, et al. Treatment of acute venous thromboembolism with dabigatran or warfarin and pooled analysis. Circulation. 2014;129(7):764-772.

21. Schulman S, Kearon C, Kearon C, et al. Extended use of dabigatran, warfarin, or placebo in venous thromboembolism. N Engl J Med. 2013; 368(8):709-718.

22. EINSTEIN Investigators. Oral rivaroxaban for symptomatic venous thromboembolism. N Engl J Med. 2010;363(26):2499-2510.

23. EINSTEIN-PE Investigators. Oral rivaroxaban for the treatment of symptomatic pulmonary embolism. $N$ Engl J Med. 2012;366(14): 1287-1297.

24. Agnelli G, Buller HR, Cohen A, et al. Oral apixaban for the treatment of acute venous thromboembolism. N Engl J Med. 2013;369(9):799-808.

25. Agnelli G, Buller HR, Cohen A, et al. Apixaban for extended treatment of venous thromboembolism. N Engl J Med. 2013;368(8):699-708.

26. Schulman S, Kearon C. Subcommittee on Control of Anticoagulation of the Scientific and Standardization Committee of the International Society on Thrombosis and Haemostasis. Definition of major bleeding in clinical investigations of antihemostatic medicinal products in nonsurgical patients. J Thromb Haemost. 2005;3(4):692-694.

27. Alotaibi GS, Almodaimegh H, McMurtry MS, Wu C. Do women bleed more than men when prescribed novel oral anticoagulants for venous thromboembolism? A sex-based meta-analysis. Thromb Res. 2013; 132(2):185-189.

28. Chai-Adisaksopha C, Crowther M, Isayama T, Lim W. The impact of bleeding complications in patients receiving target-specific oral anticoagulants: a systematic review and meta-analysis. Blood. 2014;124(15): 2450-2458.

29. Rosand J, Eckman MH, Knudsen KA, Singer DE, Greenberg SM. The effect of warfarin and intensity of anticoagulation on outcome of intracerebral hemorrhage. Arch Intern Med. 2004;164(8):880-884.

30. Fang MC, Go AS, Chang Y, et al. Death and disability from warfarinassociated intracranial and extracranial hemorrhages. Am J Med. 2007; 120(8):700-705.

31. Connolly SJ, Ezekowitz MD, Yusuf S, et al. Dabigatran versus warfarin in patients with atrial fibrillation. $N$ Engl J Med. 2009;361(12): 1139-1151.

32. Patel MR, Mahaffey KW, Garg J, et al. Rivaroxaban versus warfarin in nonvalvular atrial fibrillation. N Engl J Med. 2011;365(10):883-891.

33. Granger CB, Alexander JH, McMurray JJV, et al. Apixaban versus warfarin in patients with atrial fibrillation. N Engl J Med. 2011;365(11): 981-992.

34. Holster IL, Valkhoff VE, Kuipers EJ, Tjwa ET. New oral anticoagulants increase risk for gastrointestinal bleeding: a systematic review and meta-analysis. Gastroenterology. 2013;145(1):105-112.e15. 
35. Schiele F, van Ryn J, Canada K, et al. A specific antidote for dabigatran: functional and structural characterization. Blood. 2013;121(18): 3554-3562.

36. Glund S, Stangier J, Schmohl M, et al. Abstract 17765: a specific antidote for dabigatran: immediate, complete and sustained reversal of dabigatran induced anticoagulation in healthy male volunteers. Circulation. 2013;128:A17765.

37. Glund S, Stangier J, Schmohl M, et al. Idarucizumab, a specific antidote for dabigatran: immediate, complete and sustained reversal of dabigatran induced anticoagulation in elderly and renally impaired subjects. Poster presented at: 56th American Society of Hematology Annual Meeting and Exposition; December 6-9; 2014; San Francisco, CA

38. Boehringer Ingelheim $\mathrm{GmbH}$. Boehringer Ingelheim submits applications for approval of idarucizumab*, specific reversal agent to dabigatran etexilate $\left(\operatorname{Pradaxa}^{\mathbb{\mathbb { }}}\right)$, to EMA, FDA and Health Canada [press release]. Ingelheim am Rhein: Boehringer Ingelheim $\mathrm{GmbH}$; 2015 [March 3]. Available from http://www.boehringer-ingelheim. com/news/news_releases/press_releases/2015/03_march_2015_ dabigatranetexilate.html. Accessed July 6, 2015.

39. Bytzer P, Connolly SJ, Yang S, et al. Analysis of upper gastrointestinal adverse events among patients given dabigatran in the RE-LY trial. Clin Gastroenterol Hepatol. 2013;11(3):246-252.e1-e5.

40. Uchino K, Hernandez AV. Dabigatran association with higher risk of acute coronary events: meta-analysis of noninferiority randomized controlled trials. Arch Intern Med. 2012;172(5):397-402.

41. Giglio AF, Basile E, Santangeli P, Di Biase L, Trotta F, Natale A. Increased risk of myocardial infarction with dabigatran. $J$ Cardiovasc Med. 2014;15(1):19-26.

42. Hurlen M, Abdelnoor M, Smith P, Erikssen J, Arnesen H. Warfarin, aspirin, or both after myocardial infarction. NEngl J Med. 2002;347(13): 969-974.

43. Oldgren J, Budaj A, Granger CB, et al. Dabigatran vs placebo in patients with acute coronary syndromes on dual antiplatelet therapy: a randomized, double-blind, phase II trial. Eur Heart J. 2011;32(22): 2781-2789.
44. Mega JL, Braunwald E, Wiviott SD, et al. Rivaroxaban in patients with a recent acute coronary syndrome. N Engl J Med. 2012;366(1):9-19.

45. Alexander JH, Lopes RD, James S, et al. Apixaban with antiplatelet therapy after acute coronary syndrome. N Engl J Med. 2011;365(8):699-708.

46. Ageno W. Oral anticoagulant therapy. Chest. 2012;141(2 Suppl):e44S.

47. Awad NI, Brunetti L, Juurlink DN. Enhanced elimination of dabigatran through extracorporeal methods. J Med Toxicol. 2015;11(1):85-95.

48. Alikhan R, Rayment R, Keeling D, et al. The acute management of haemorrhage, surgery and overdose in patients receiving dabigatran. Emerg Med J. 2014;31(2):163-168.

49. Reilly PA, Lehr T, Haertter S, et al. The effect of dabigatran plasma concentrations and patient characteristics on the frequency of ischemic stroke and major bleeding in atrial fibrillation patients: the RE-LY Trial (Randomized Evaluation of Long-Term Anticoagulation Therapy). J Am Coll Cardiol. 2014;63(4):321-328.

50. MacLean S, Mulla S, Akl EA, et al. Patient values and preferences in decision making for antithrombotic therapy: a systematic review: Antithrombotic Therapy and Prevention of Thrombosis, 9th ed: American College of Chest Physicians Evidence-Based Clinical Practice Guidelines. Chest. 2012;141(2 Suppl):e1S-e23S.

51. Piaggio G, Elbourne DR, Pocock SJ, Evans SJW, Altman DG; CONSORT Group. Reporting of noninferiority and equivalence randomized trials: extension of the CONSORT 2010 statement. JAMA. 2012; 308(24):2594-2604.

52. Piaggio G, Elbourne DR, Altman DG, Pocock SJ, Evans SJW; CONSORT Group. Reporting of noninferiority and equivalence randomized trials: an extension of the CONSORT statement. JAMA. 2006;295(10): 1152-1160.

53. Eerenberg ES, Kamphuisen PW, Sijpkens MK, Meijers JC, Buller HR, Levi M. Reversal of rivaroxaban and dabigatran by prothrombin complex concentrate: a randomized, placebo-controlled, crossover study in healthy subjects. Circulation. 2011;124(14):1573-1579.

54. Vedovati MC, Germini F, Agnelli G, Becattini C. Direct oral anticoagulants in patients with VTE and cancer: a systematic review and meta-analysis. Chest. 2015;147(2):475-483.

\section{Publish your work in this journal}

Drug Design, Development and Therapy is an international, peerreviewed open-access journal that spans the spectrum of drug design and development through to clinical applications. Clinical outcomes, patient safety, and programs for the development and effective, safe, and sustained use of medicines are a feature of the journal, which

\section{Dovepress}

has also been accepted for indexing on PubMed Central. The manuscript management system is completely online and includes a very quick and fair peer-review system, which is all easy to use. Visit http://www.dovepress.com/testimonials.php to read real quotes from published authors. 